\title{
La Mecanique En Classe De Seconde Scientifique : Analyses Des Styles Pedagogiques Et Des Documents Iconographiques Dans Le Manuel Intitule Physique Chimie (Collection Arex), En Usage Actuel En Cote d'Ivoire
}

\section{Kouamé Nguessan}

Laboratoire interdisciplinaire de didactique des disciplines et de psychologie de l'éducation, Département des Sciences de l'éducation/Ecole Normale Supérieure d'Abidjan

doi: 10.19044/esj.2016.v12n19p354 URL:http://dx.doi.org/10.19044/esj.2016.v12n19p354

\begin{abstract}
This contribution sets out, through the analysis of the teaching styles, the texts and graphic material of the six chapters about Mechanics of the handbook Physique Chimie (Collection AREX, Les classiques africains) of the first years of high school science classes, to highlight the difficulties and obstacles that the teacher or the student face in using it.. The results of the analysis from the epistemological and didactic benchmarks knowledge to teach the mechanics show that the manual emphasizes the use of more informative teaching style and that of the illustrative figures to state the knowledge to be taught. Thus, the approaches used do not refer to a real problematization and do not allow students to be independent and productive; they are not actively and intellectually engage students in the learning process.

Faced with the passivity of the student in the acquisition of knowledge, it thus seems necessary to include in the training of the teacher, a teacher training course based on understanding of texts and teaching styles to support a constructivist pedagogy class.
\end{abstract}

Keywords: Mechanical, teaching styles, iconographic documents, manual, first year high school science classes, difficulty.

\section{Resume}

Cette contribution se propose, à travers l'analyse des styles pédagogiques, des documents textuels et iconographiques des six chapitres de Mécanique du manuel scolaire intitulé Physique Chimie (Collection AREX, Les classiques africains) des classes de seconde scientifique, de 
mettre en évidence les difficultés et les obstacles auxquels l'enseignant ou l'élève se trouve confronté dans son utilisation. Les résultats de l'analyse faite à partir des repères épistémologique et didactique du savoir à enseigner de la Mécanique, montrent que le manuel privilégie plus l'usage du style pédagogique informatif et celui des figures illustratives pour énoncer le savoir à enseigner. Ainsi, les démarches utilisées ne font pas référence à une vraie problématisation et ne permettent pas à l'élève d'être autonome et productif ; elles n'engagent pas activement et intellectuellement l'élève dans le processus d'apprentissage.

Face à la passivité de l'élève dans l'acquisition des connaissances, il parait donc nécessaire d’intégrer dans la formation continue de l'enseignant, une formation didactique basée sur la compréhension des textes et des styles pédagogiques pour soutenir une pédagogie constructiviste en classe.

Mots clés : Mécanique, styles pédagogiques, documents iconographiques, manuel, seconde scientifique, difficulté.

\section{INTRODUCTION}

Quel enseignant de Sciences Physiques n’a-t-il pas entendu les élèves dire que la Mécanique est trop mathématisée, donc difficile à comprendre ?

Toutes les analyses convergent: l’apprentissage de la Mécanique exige la synergie de compétences multiples, langagières, symboliques, mathématiques (Hannoun, 2008). Cette partie de la physique est perçue, de manière presque universelle, comme synonyme de formules mathématiques et de résultats à mémoriser.

L’enseignement de la Mécanique au lycée occupe une place importance dans la construction/acquisition des connaissances chez les élèves. Ainsi, cette partie occupe dans le programme actuel de l'enseignement de physique et chimie en classe de seconde scientifique en Côte d'Ivoire, 36 heures des 66 heures consacrées à la Physique, soit 55\% du volume horaire annuel de cette partie de la physique.

A l'enseignement secondaire, nous notons une gestion difficile des programmes et du système d'évaluation due aux conditions dans lesquelles se développe le processus d'apprentissage : effectif pléthorique dans les classes, manque de matériel didactique, programme qui n’intègre pas la réalité nationale.

Pour pallier aux énormes difficultés aussi bien au niveau des programmes d'enseignement qu'au niveau du personnel enseignant, le programme de Mécanique a subi plusieurs réformes ayant pour objectif de répondre efficacement aux exigences de la modernisation et aux défis de la mondialisation. Ainsi, le suivi rapproché de la gestion des programmes, du 
système d’évaluation et des informations focalisées dans les besoins, ont été des moyens de résolution des problèmes curriculaires.

Pour prendre en compte les objectifs généraux : apprendre à définir un système, comprendre et savoir utiliser le concept de force et le principe de l'inertie, savoir effectuer un "bilan de forces" du programme de Mécanique de la classe de seconde scientifique, nous avons assisté à l'introduction des nouvelles technologies d'information et de communication comme support au processus d'apprentissage en vue de dépasser les problèmes liés à la rareté des matériels didactiques, et améliorer la qualité de l’enseignement secondaire.

Le manuel scolaire de Physique Chimie, collection « AREX ${ }^{1}$ » est conçu en conséquence pour tenir compte du programme révisé de sciences physiques, et il est recommandé comme seul manuel en usage dans l'enseignement secondaire en Côte d'Ivoire depuis 2000. Ce manuel devrait faire la synthèse entre le fondamental et l'appliqué, le permanent et l'évolutif, le stable et l'innovant, la reproduction et la création, en vue de donner des outils nécessaires et utilisables par les élèves dans la résolution des problèmes techniques ou pratiques auxquels ils sont confrontés dans la vie quotidienne.

Le défi de ce travail est de faire une analyse critique des styles pédagogiques et les documents iconographiques de la partie Mécanique du manuel dans le but de comprendre leur efficacité dans l'enseignement/apprentissage de la mécanique en classe de seconde scientifique. Les analyses sont centrées essentiellement sur les caractéristiques des styles pédagogiques et des figures, utilisés dans cette partie du manuel.

Ainsi, dans un premier temps, nous allons définir les repères didactique et épistémologique ; dans un deuxième temps, nous préciserons la problématique suivie de la méthodologie; dans un troisième et dernier temps, nous présenterons les résultats et leur discussion.

\section{METHODES ET MATERIELS}

\section{Quelques repères didactique et épistémologique}

\section{Le modèle KVP}

La didactique d'une discipline est classiquement centrée sur le contenu de ce qui est enseigné et appris dans cette discipline. Une analyse didactique des manuels scolaires s’intéresse ainsi au contenu de ces manuels, mais elle cherche aussi à rendre compte de leurs messages, qu'ils soient explicites ou implicites.

\footnotetext{
${ }^{1}$ Atelier de Recherche pour un Enseignement EXpérimental des sciences physiques
} 
Le contenu scientifique et technique est explicitement en interaction avec les pôles que sont : les pratiques sociales (P), les systèmes de valeurs (V), et les connaissances scientifiques (K).

Ce modèle (Clément, 1998, 2004) considère l'existence d'une interaction entre trois pôles, indiquée selon la figure 1 ci-après :

Figure 1 Triangle d'interaction entre les connaissances scientifiques (K), les systèmes de valeurs $(V)$ et les pratiques sociales $(P)$

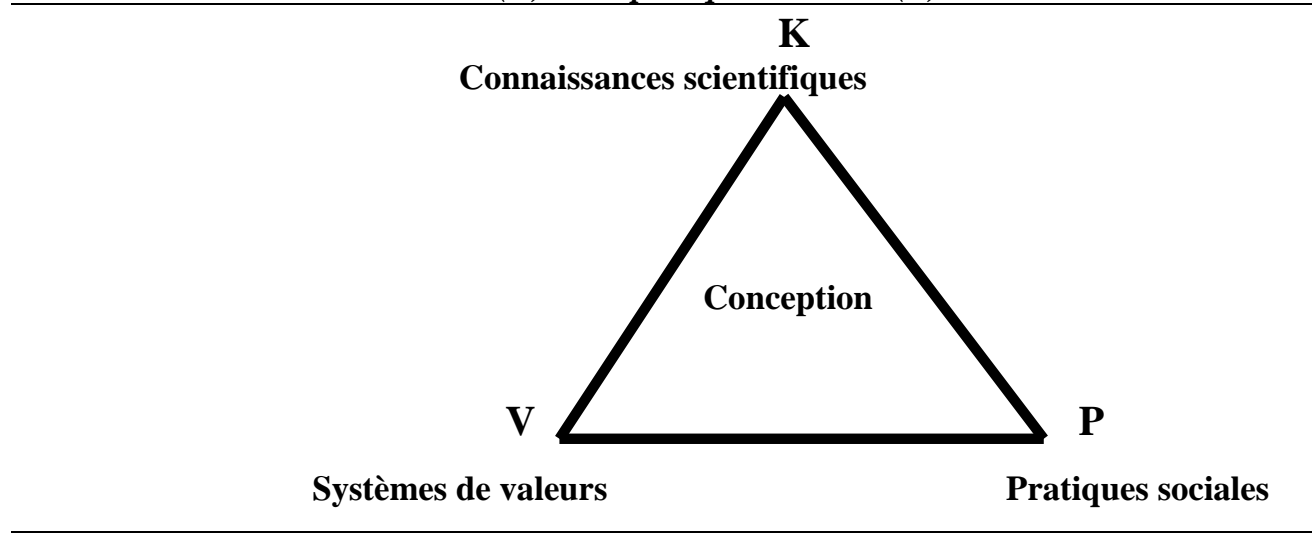

Source : Modèle de Clément 1998, 2004

Selon Bernard, Clément \& Carvalho (2007), analyser ces interactions KVP à partir des contenus des manuels nécessite :

o des connaissances dans le domaine disciplinaire (la Mécanique);

o une distance épistémologique critique par rapport à ces contenus disciplinaires ;

o une approche historique aidant à identifier des obstacles épistémologiques, les limites, voire les erreurs, de nos connaissances passées ;

0 une culture dans la didactique (de la physique);

o une analyse anthropologique du contexte socioéconomique et culturel lié aux pratiques sociales et valeurs dominantes qui interfèrent avec ces connaissances.

\section{Les styles pédagogiques}

L'axe d'analyse relatif aux styles pédagogiques est construit de façon à permettre de répertorier les styles d'approches préconisés par les éditeurs de manuels. Cet axe d'analyse présente quatre styles pédagogiques visant à catégoriser les contenus textuels. Chaque phrase peut être référencée suivant l'un des styles en fonction du degré de la problématisation, de la participation et du développement de l'esprit critique de l'élève. La figure 2 présente les quatre styles pédagogiques et leurs indicateurs. 
Figure 2 Grille des quatre styles pédagogiques et de leurs indicateurs

STYLE PEDAGOGIGUE

STYLE ET STRUCTURE DES

PHRASES

INFORMATIF

Descriptif, informatif : phrases avec des faits reconnus,

des données

INJONCTIF

Impératif, injonctif :

"Vous devez faire ceci..., faites que... parce que c'est comme

ça, parce que

religion

c’est la manière..., parce que la science le montre ..., parce que la dit que ..."

PERSUASIF

Persuasif :

ceci..., faites que..."

"Vous pouvez conclure cela..., vous devrez (ou devriez) faire

dérangement sérieux

"Voyez les avantages de prendre cette solution... ou le de ne pas prendre cette solution..."

PARTICIPATIF

proposées.

\section{Participatif :}

Présentant plusieurs points de vue. Plusieurs solutions sont Demander les idées des élèves, leurs suggestions...

\section{Les différents types de texte}

La figure 3 qualifie le type de texte et donne ses caractéristiques, ce que Lasswell (1952) appelle sa «teneur " sa valeur informationnelle, les mots, les idées, les arguments, les conclusions, les affirmations-négations, bref ce qu'on a l'habitude d'appeler littéralement le « contenu ».

Figure 3 Grille des six types de texte et leurs caractéristiques

TYPE DE TEXTE CARACTERISTIQUES

NARRATIF $\quad$ Texte structuré qui raconte une histoire réelle ou imaginaire

DESCRIPTIF

Texte qui décrit une expérience, une image, un événement, etc.

INFORMATIF

Texte qui énonce des faits réels, vérifiables dont le but est

d'informer

PRESCRIPTIF Texte présenté de façon très structurée et codifiée. On le trouve dans les recettes, modes d'emploi, notices, protocoles expérimentaux, etc.

ARGUMENTATIF Texte qui cherche à convaincre le lecteur.

EXPLICATIF Texte qui vise à expliquer un énoncé, un fait, un phénomène. 
- Les documents iconographiques

L'iconographie est un ensemble où texte et illustration sont étroitement imbriqués : chaque photographie et chaque dessin s'intègre à sa meilleure place pour donner une efficacité pédagogique au manuel.

Le document iconographique, message virtuel, il a pour rôle d'informer, d'illustrer, d'expliquer, d'attirer le lecteur, de faire participer l'élève.

Figure 4 Grille des caractéristiques des documents iconographiques

DOCUMENTS ICONOGRAPHIQUES CARACTERISTIQUES

IMAGE Une représentation visuelle d'un objet, d'un être vivant ou d'un concept. Elle peut être informative si elle apporte de nouvelles connaissances; illustrative si elle met en valeur et embellit le sujet ou les pages du manuel; explicative si elle permet d'éclaircir une notion ; participative si elle entraîne un travail de la part des élèves.

Par rapport au texte, l'image peut être supplémentaire (elle apporte des informations ou des explications en plus par rapport au texte) ; l'image peut être complémentaire (elle complète le texte sans apporter d'informations supplémentaires); l'image peut être incluse dans le texte lorsque celui-ci en fait référence.

DESSIN Une simplification et une mise en évidence de ce qui est essentiel. C’est une représentation aussi exacte que possible de la réalité, respectant forme, proportions, et détails. Il doit être comparable à une photo.

SCHEMA

Une représentation des éléments essentiels d'un seul coup et ne sont pas forcément présentés tels qu’on les voit dans la réalité. C'est une représentation non ressemblante d'un objet ou d'une idée, d'un phénomène. Il peut utiliser couleurs, codes, symboles... Le schéma est parfois extrêmement codifié.

PHOTOGRAPHIE Elle contribue à apporter des informations les plus proches de la réalité possible.

Dans le manuel intitulé Physique Chimie de la collection "AREX» des classes de seconde scientifique, nous avons deux types de documents iconographiques qui sont: les figures (les images, les dessins et les schémas), et la photographie.

\section{Problématique}

Basé sur le programme révisé de sciences physiques en vigueur en Côte d'Ivoire depuis octobre 2000, le manuel intitulé Physique Chimie de la collection "AREX » de la classe de seconde scientifique, comporte trois modules : module de Mécanique, module d'Electricité, et module de Chimie. Chaque module comprend trois grandes parties : 
- Une première partie, nommée phase d’acquisition de connaissances, subdivisée en quatre rubriques: le cours présenté sur des pages illustrées; un résumé terminant chaque cours sous la rubrique «L’essentiel »; une activité expérimentale où sont présentés les protocoles expérimentaux ; et une fiche « Méthode » qui s’adresse particulièrement aux élèves et a pour but d'initier ces derniers au maniement de l'outil mathématique et à la pratique du matériel scientifique adapté au contexte africain.

- Une deuxième partie, appelée phase d’évaluation des connaissances, subdivisée en deux rubriques: l'une pour la fixation des connaissances, intitulée «Vérifions les connaissances »; et l’autre pour leur consolidation, intitulée « Appliquons nos connaissances ».

- Une troisième et dernière partie, nommée activité documentaire pour aider à élargir le champ de réflexion de l'élève au-delà du minimum exigible, en vue d'accroître la culture scientifique et technique de ce dernier.

Le manuel est donc conçu à l’aide des instruments sélectionnés pour faciliter son utilisation. Ces instruments contribuent malheureusement aux inégalités scolaires lorsque l'auteur, pas toujours conscient des difficultés des élèves, considère leur usage comme transparent et allant de soi. C'est en ce sens que l'analyse de ces instruments ou supports didactiques est non seulement essentielle mais fondamentale surtout en considérant les difficultés constatées au niveau des élèves dans leur utilisation.

Les évolutions récemment apportées au programme d’enseignement de la Mécanique, donc du manuel actuellement en usage ont comme but de susciter l'intérêt des élèves, et d’obtenir leur adhésion sincère, indépendamment du rôle que joue cette partie de la physique dans la réussite scolaire. Malgré cela, nous constatons le manque d'engouement de ces derniers dans l'utilisation du manuel pour acquérir les connaissances. L’enseignant reste incontestablement la principale source du savoir. Ainsi, la partie Mécanique du manuel intitulé Physique Chimie de la collection « AREX » de la classe de seconde scientifique ne semble pas les passionner, encore moins soulever leur enthousiasme. Pourquoi ce désintérêt pour l'usage du manuel de physique par les élèves de seconde scientifique ? Les styles pédagogiques adoptés et les documents utilisés dans le manuel par l'auteur permettent-ils aux élèves de construire leur savoir ?

Ces interrogations sont des exemples de l'exigence légitime à vouloir comprendre l'intérêt d'apprendre, c'est-à-dire à donner un sens à l'apprentissage.

La question de recherche qui découle du désintérêt des élèves de seconde scientifique pour l'utilisation de leur manuel est la suivante : quel est l'impact des styles pédagogiques et les documents iconographiques 
utilisés dans le manuel sur la compréhension du savoir à enseigner relatif à la Mécanique ? Cette question comporte deux aspects : le premier se focalise sur l'analyse des documents textuels et iconographiques qui sont utilisés pour construire le savoir à enseigner relatif à la Mécanique alors que le deuxième vise à identifier les difficultés, les obstacles dans l'utilisation du manuel intitulé Physique Chimie de la collection «AREX » de la classe de seconde scientifique.

\section{Matériels}

Le matériel de travail est constitué du contenu du savoir à enseigner de la partie Mécanique de physique du manuel scolaire intitulé Physique Chimie (Collection AREX, Les classiques africains) des classes de seconde scientifique. Cette partie concerne six chapitres. Le critère de choix est relatif à la présence de thèmes en rapport avec la Mécanique. Les thèmes identifiés sont : Le mouvement, Actions mécaniques, Equilibre d'un solide soumis à deux forces ou à trois forces non parallèles, Equilibre d'un solide mobile autour d'un axe fixe, Principe de l'inertie, Quantité de mouvement.

La population est constituée de 54 étudiants, niveau Master Professionnel en physique et chimie, en formation professionnelle à l'Ecole Normale Supérieure (ENS) d'Abidjan au cours de l'année scolaire 2015/2016. Ces 54 étudiants sont choisis aléatoirement parmi 72. Avec ces 54 étudiants, nous avons constitué 3 groupes de travail notés $\mathrm{A}, \mathrm{B}$, et $\mathrm{C}$. En rapport avec les 6 chapitres de Mécanique, nous avons subdivisé chaque groupe en 3 sous groupes; soit 9 sous groupes au total notés : $A_{1}, A_{2}, A_{3}, B_{1}$, $B_{2}, B_{3} ; C_{1}, C_{2}, C_{3}$. Chaque sous groupe est invité à travailler sur un chapitre. Ainsi pour un chapitre donné, 3 sous groupes y ont travaillé.

\section{Méthodes}

Trois étapes ont été suivies :

D’abord, à partir des repères épistémologique et didactique, et le savoir à enseigner relatif à la Mécanique de la classe de seconde scientifique, deux grilles d'analyse qualitative ont été élaborées : l'une est relative à l'analyse des styles pédagogiques adoptés par les auteurs dans le manuel et l'autre fait référence à l'analyse des documents iconographiques du manuel.

Ensuite, le travail est validé par groupe et par salle, soit 3 salles retenues pour le travail.

Enfin, après les avoir validés dans les différents groupes, nous nous sommes retrouvés tous au laboratoire pour harmoniser les points de vue sur chaque chapitre identifié.

\section{RESULTATS}


Les données recueillies ont permis de renseigner les différentes figures issues des deux grilles ci-dessus mentionnées. Les deux premières enregistrées (figures 5 et 6) mentionnent les caractéristiques des différents styles pédagogiques utilisés au niveau du savoir à enseigner des différents chapitres de Mécanique de la seconde scientifique. Les figures 7, 7.1, 7.2, 7.3, 7.4 répertorient les proportions de l'aspect qualitatif des documents textuels et iconographiques du manuel.

\section{Résultats relatifs aux styles pédagogiques}

Les deux figures (5 et 6) rendent compte les caractéristiques des styles pédagogiques issus du manuel intitulé Physique Chimie, collection AREX, de la classe de seconde scientifique de la partie Mécanique. du manuel.

La figure 5 répertorie par chapitre les différents styles pédagogiques 
Figure 5 Caractéristiques des différents styles pédagogiques utilisés au niveau du savoir à enseigner en Mécanique en classe de seconde scientifique par chapitre

\begin{tabular}{|c|c|c|c|c|c|}
\hline \multirow{3}{*}{ Chapitre } & & \multirow{2}{*}{\multicolumn{4}{|c|}{ Styles pédagogiques }} \\
\hline & & & & & \\
\hline & & Style informatif & \multirow[t]{2}{*}{ Style injonctif } & \multirow[t]{2}{*}{ Style persuasif } & Style participatif \\
\hline \multicolumn{3}{|c|}{ Le mouvement } & & & \\
\hline 1. & Caractère relatif du mouvement & + & & & \\
\hline \multirow[t]{2}{*}{2.} & Vitesse & + & & & \\
\hline & Fiche méthode & + & + & & + \\
\hline \multicolumn{6}{|c|}{ Actions mécaniques } \\
\hline 1. & Effets d'une action mécanique & + & + & & \\
\hline 2. & Modélisation d'une force & + & & & \\
\hline 3. & Etude de quelques forces & + & & & + \\
\hline \multirow[t]{2}{*}{4.} & Principe des actions réciproques & + & + & & + \\
\hline & Activité expérimentale & + & + & & + \\
\hline \multicolumn{6}{|c|}{$\begin{array}{l}\text { Equilibre d'un solide soumis à deux forces ou } \\
\text { à trois forces non parallèles }\end{array}$} \\
\hline 1. & Qu'est-ce qu’un système ? & + & & & \\
\hline 2. & $\begin{array}{l}\text { Inventaire des forces extérieures appliquées } \\
\text { à un système }\end{array}$ & + & & + & \\
\hline 3. & $\begin{array}{l}\text { Conditions d'équilibre d'un solide soumis } \\
\text { à deux forces }\end{array}$ & + & & & \\
\hline 4. & $\begin{array}{l}\text { Conditions d'équilibre d'un solide soumis } \\
\text { à trois forces non parallèles }\end{array}$ & + & + & & + \\
\hline \multirow[t]{2}{*}{5.} & Application & + & + & & + \\
\hline & Activité expérimentale & + & $\perp$ & & + \\
\hline
\end{tabular}




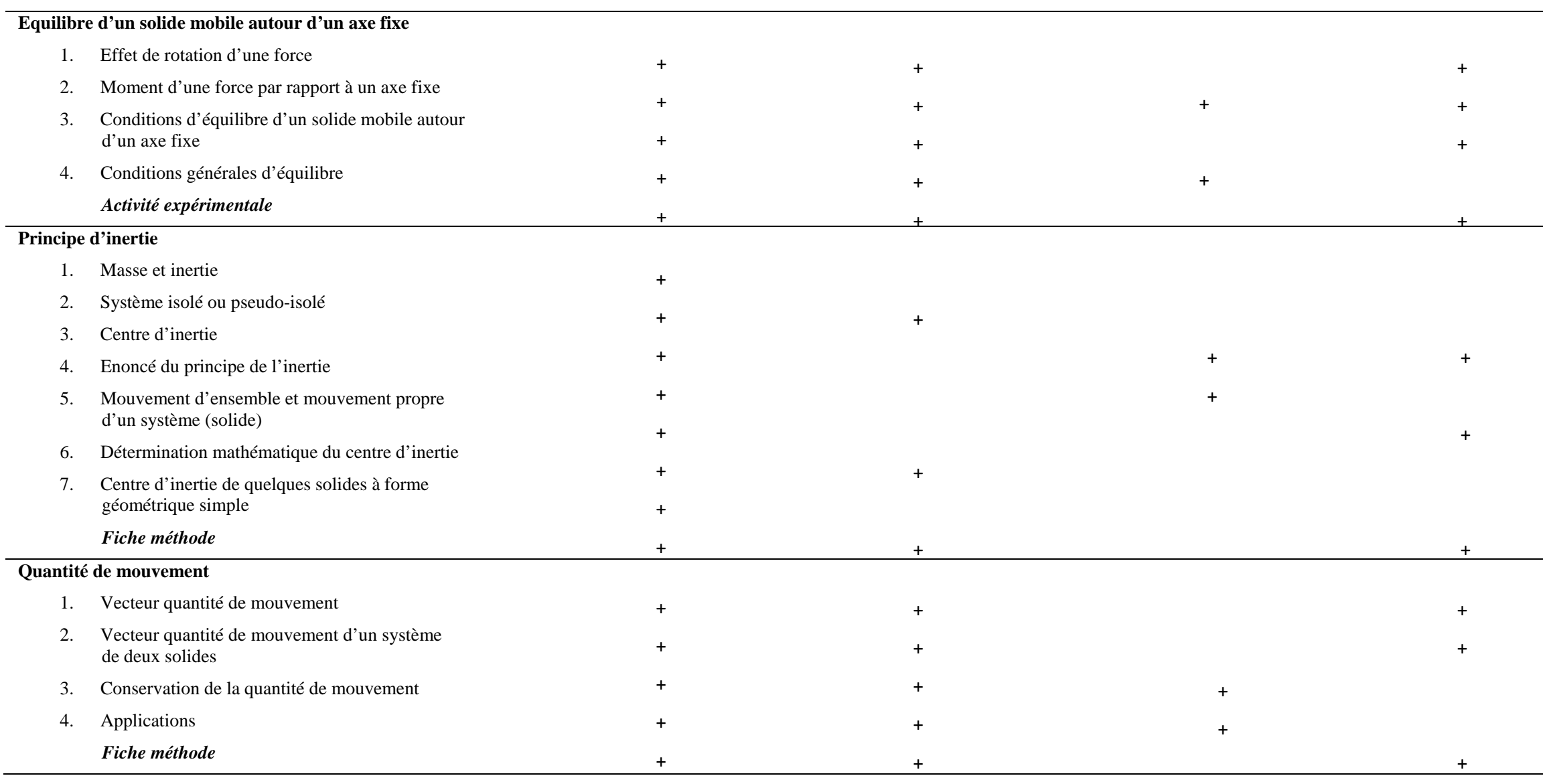


Nous regroupons en pourcentage, les quatre styles pédagogiques utilisés dans le manuel sur la figure 6 ci-après :

Figure 6 Récapitulatif de la typologie des styles pédagogiques dans le manuel AREX, Mécanique, seconde scientifique

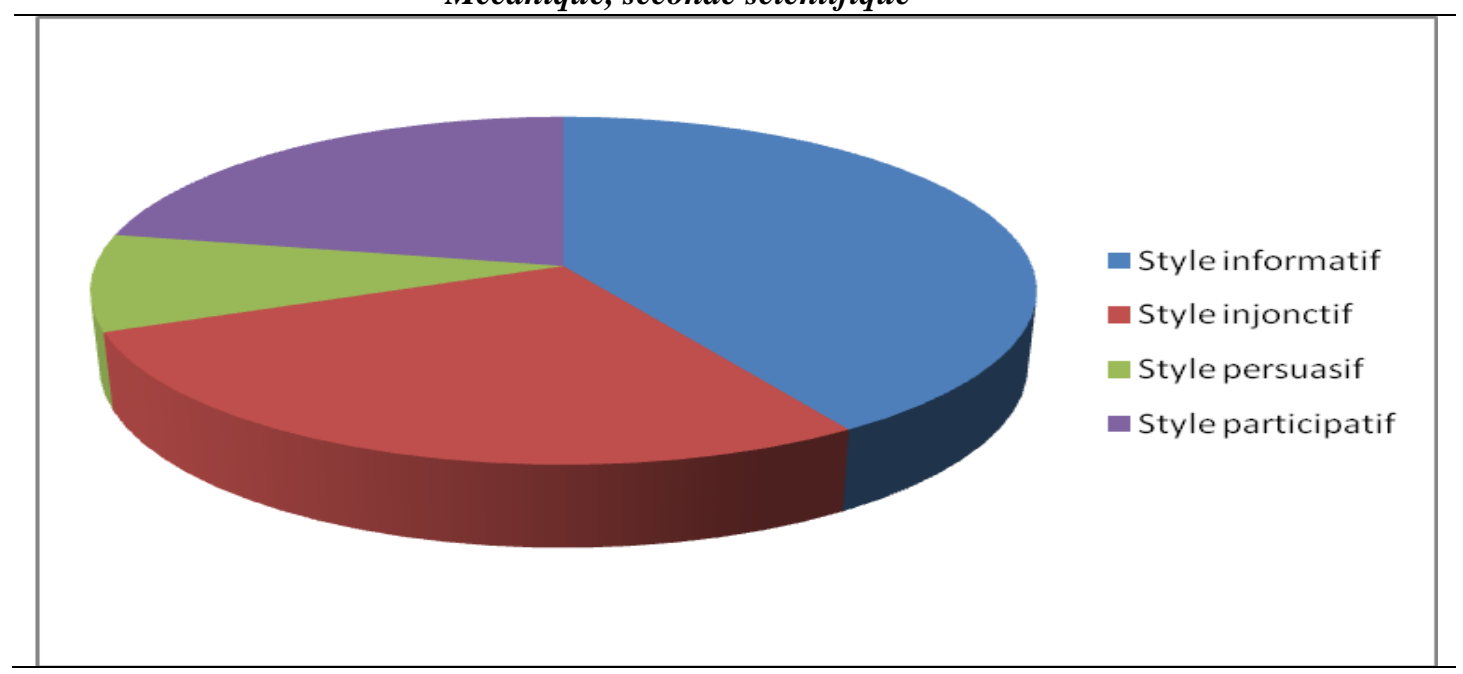

La figure 6 répertorie en pourcentage la proportion de chaque style pédagogique dans le manuel intitulé Physique Chimie de la collection AREX de la classe de seconde scientifique.

Ainsi, nous notons que le style pédagogique informatif est le plus utilisé avec $41 \%$, suivi du style pédagogique injonctif avec $28 \%$; puis du style pédagogique participatif avec $22 \%$; et enfin, du style pédagogique persuasif avec $9 \%$.

Le style pédagogique informatif prédomine. L’importance que les auteurs lui accordent à travers le manuel traduit une faible problématisation et une très faible incitation à l'autonomie et à la réflexion. Cela ne développe pas chez l'apprenant le plaisir de l'invention. Aucune réflexion n'est demandée, toutes les informations sur les différentes notions issues de la Mécanique sont mises à sa disposition. En conséquence, l'élève n'est pas engagé dans l'élaboration et la construction de son savoir.

Le style pédagogique injonctif relevé à travers les différentes activités indique que les solutions sont imposées sans argumentation. L'information est transmise de manière dogmatique avec des consignes ou des directives à appliquer.

Les deux styles largement utilisés indiquent que le manuel ne semble pas être très accessible aux élèves mais plutôt destiné à l'enseignant.

\section{Résultats relatifs à l'aspect qualitatif des documents}

La figure 7 fait le point de l'aspect qualitatif des documents textuels et iconographiques dans le manuel : 
Figure 7 Aspect qualitatif des documents textuels et iconographiques du manuel de Physique Chimie classe de seconde scientifique

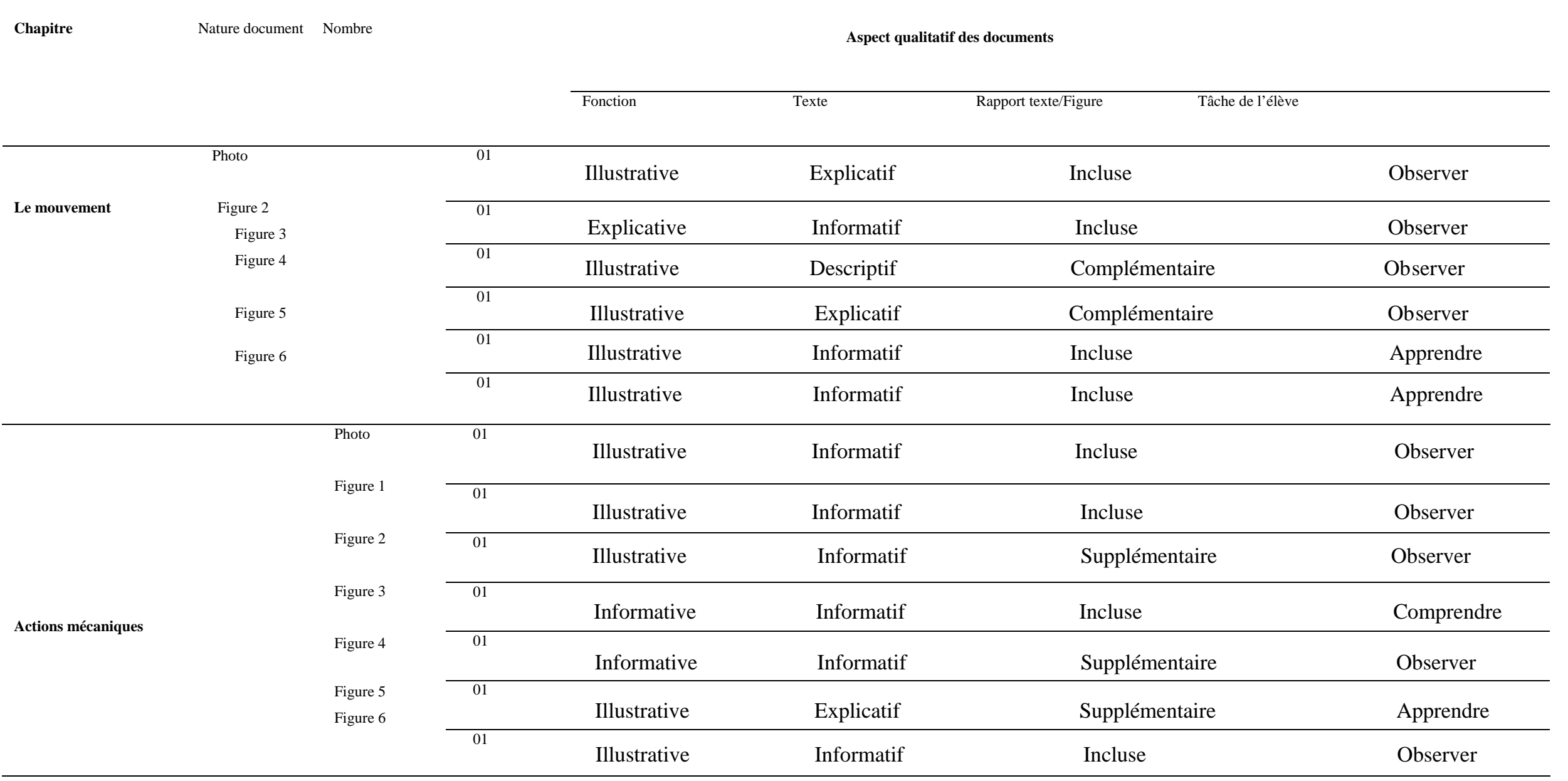




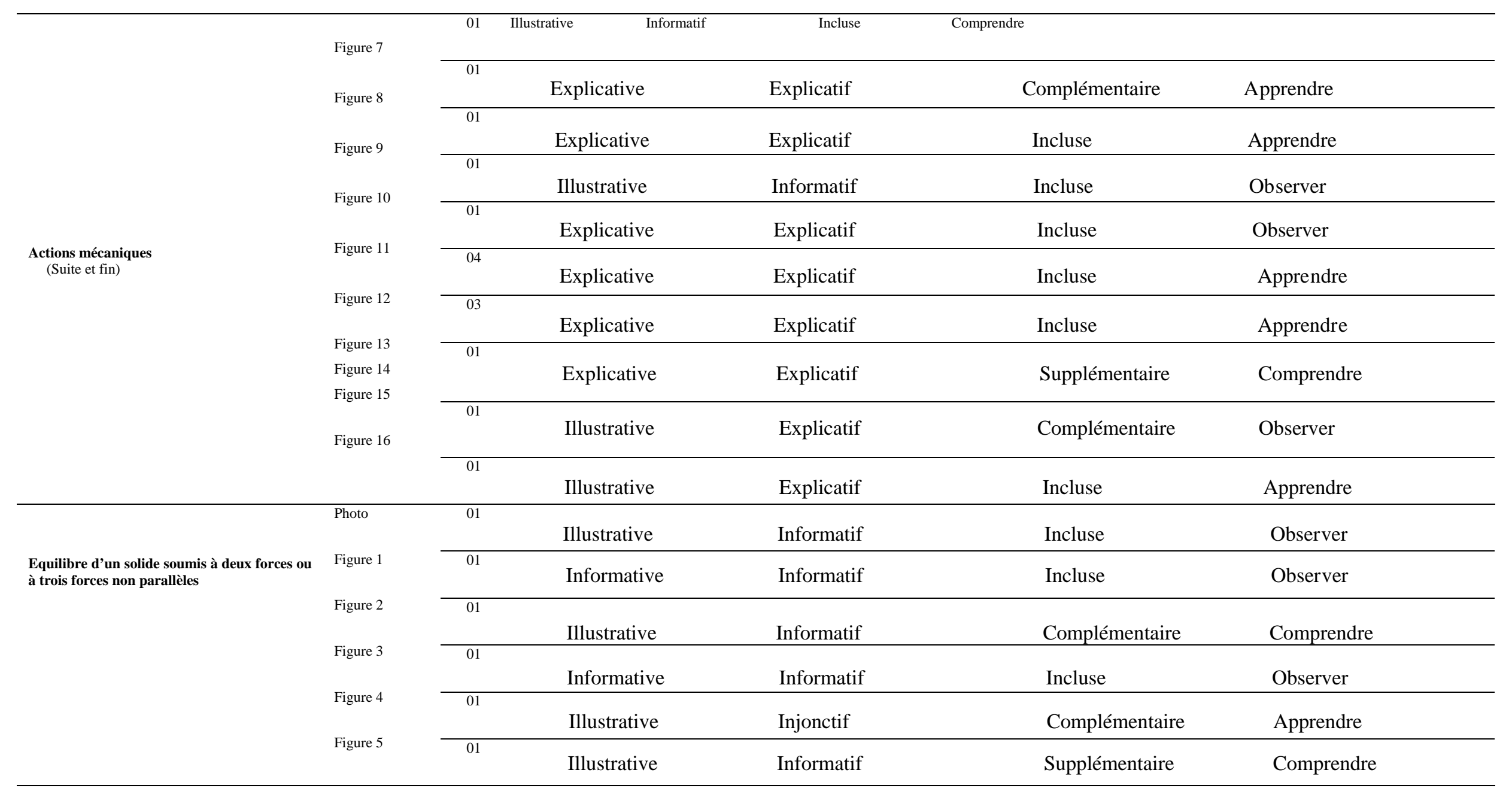




\begin{tabular}{|c|c|c|c|c|c|c|}
\hline \multirow{10}{*}{$\begin{array}{l}\text { Equilibre d'un solide soumis à deux forces ou } \\
\text { à trois forces non paralleles (Suite et fin) }\end{array}$} & $\begin{array}{l}\text { Figure } 6 \\
\end{array}$ & 01 & Illustrative & Complémentaire & Comprendre & \\
\hline & Figure 7 & 01 & Illustrative & Informatif & Complémentaire & Comprendre \\
\hline & Figure 8 & 01 & Illustrative & Informatif & Supplémentaire & Apprendre \\
\hline & Figure 9 & 01 & Informative & Informatif & Supplémentaire & Apprendre \\
\hline & Figure 10 & 01 & Illustrative & Informatif & Incluse & Comprendre \\
\hline & Figure 11 & 01 & Participative & Participatif & Incluse & Observer \\
\hline & Figure 12 & 01 & Illustrative & Informatif & Incluse & Apprendre \\
\hline & Figure 13 & 01 & Participative & Participatif & Supplémentaire & Apprendre \\
\hline & Figure 14 & 01 & Participative & Participatif & Supplémentaire & Apprendre \\
\hline & Figure 15 & 01 & Illustrative & Informatif & Supplémentaire & Apprendre \\
\hline \multirow{6}{*}{$\begin{array}{l}\text { Equilibre d'un solide mobile autour d'un axe } \\
\text { fixe }\end{array}$} & Photo & 01 & Illustrative & Informatif & Incluse & Observer \\
\hline & Figure 1 & 01 & Injonctive & Injonctif & Supplémentaire & Comprendre \\
\hline & Figure 2 & 01 & Injonctive & Injonctif & Supplémentaire & Comprendre \\
\hline & Figure 3 & 01 & Injonctive & Informatif & Supplémentaire & Apprendre \\
\hline & Figure 4 & 01 & Injonctive & Informatif & Supplémentaire & Apprendre \\
\hline & Photo & 01 & Illustrative & Informatif & Incluse & Observer \\
\hline
\end{tabular}




\begin{tabular}{|c|c|c|c|c|c|c|}
\hline \multirow{6}{*}{ Principe d'inertie } & Figure 1 & 01 & Informative & Supplémentaire & Observer & \\
\hline & Figure 2 & 01 & Illustrative & Informatif & Incluse & Comprendre \\
\hline & Figure 3 & 01 & Illustrative & Informatif & Supplémentaire & Observer \\
\hline & Figure 4 & 01 & Illustrative & Informatif & Supplémentaire & Observer \\
\hline & Figure 5 & 01 & Illustrative & Injonctif & Incluse & Comprendre \\
\hline & Figure 6 & 01 & Illustrative & Informatif & Supplémentaire & Comprendre \\
\hline \multirow{6}{*}{ Quantité de mouvement } & Photos & 01 & Illustrative & Informatif & Supplémentaire & Observer \\
\hline & Figure 1 & 01 & Illustrative & Informatif & Supplémentaire & Observer \\
\hline & & 01 & Participative & Injonctif & Incluse & Comprendre \\
\hline & 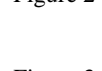 & 01 & Injonctive & Injonctif & Supplémentaire & Comprendre \\
\hline & Figure 4 & 01 & Illustrative & Informatif & Supplémentaire & Observer \\
\hline & Figure 5 & 01 & Illustrative & Informatif & Supplémentaire & Apprendre \\
\hline
\end{tabular}


Nous regroupons au niveau des figures ci-dessous par aspect qualitatif le contenu du manuel :

Figure 7.1 Classification de la fonction des documents iconographiques de la partie Mécanique

\begin{tabular}{|c|c|c|c|c|c|}
\hline \multirow{3}{*}{ Total } & \multicolumn{5}{|c|}{ Fonction } \\
\hline & Illustrative & Participative & Injonctive & Informative & Explicative \\
\hline & 35 & 4 & 5 & 6 & 7 \\
\hline
\end{tabular}

La figure 7.1 indique clairement que la plupart des documents iconographiques (6 photos et 51figures) ont une fonction illustrative. Les autres fonctions (Explicative, Informative, Injonctive et Participative) sont en proportions équivalentes, et varient de 4 à 7 . La prédominance des figures illustratives (au nombre de 35/57) indique que par le visuel, nous avons les mêmes informations aussi bien au niveau des figures que les textes qui leur sont associés ; donc pas d'informations supplémentaires apportées. Elles sont suivies par les figures à fonction participative (7/57) qui sont conçues pour éclaircir une notion donnée.

Figure 7.2 Classification du rapport Texte/Documents iconographiques dans le manuel de la partie Mécanique

\section{Rapport Texte/Figure}

Incluse

Complémentaire

Total Supplémentaire

Nous notons que sur les 57 documents iconographiques, 27 font référence aux textes, 22 apportent des informations ou des explications en plus par rapport aux textes, et 8 autres complètent les textes sans apporter d'informations supplémentaires. Ces résultats $(27+8)$ documents iconographiques confirment qu'ils n'apportent pas d'informations supplémentaires mais ils sont plutôt intégrés dans le contenu du manuel pour illustrer les textes et parfois faire comprendre certaines choses qui sont plus faciles à interpréter visuellement.

Figure 7.3 Classification du contenu des textes de la partie Mécanique

\begin{tabular}{rrrrrr}
\hline \multirow{2}{*}{ Total } & & \multicolumn{5}{c}{ Texte } \\
Explicatif & Informatif & Descriptif & Injonctif & Participatif \\
\cline { 2 - 6 } & $\mathbf{1 1}$ & 36 & $\mathbf{1}$ & $\mathbf{6}$ & $\mathbf{3}$ \\
\hline
\end{tabular}

La figure 7.3 montre que sur les 57 textes analysés, 36 sont de type informatif, 11 sont de type explicatif, 6 de type injonctif, 3 de type 
participatif, et 1 est de type descriptif. Ce qui indique que la plupart des textes énoncent des faits dont le but est d'informer. Les auteurs ont plus tendance à donner des informations à l'utilisateur du manuel que de solliciter sa participation à la compréhension et à la construction du savoir.

Figure 7.4 Classification de la tâche de l'élève dans le manuel de la partie Mécanique

Tâche de l'élève

Total

Apprendre Comprendre

Observer

18

15

24

Nous enregistrons au niveau des tâches dédiées à l'élève :

- 24 tâches pour effectuer un travail d'observation, il n'y a pas d'activités demandées à l'élève, il doit juste lire le document iconographique ;

- 18 tâches pour apprendre de nouvelles connaissances à l'aide des documents iconographiques, il s’agit d'engendrer chez l'élève une accumulation de connaissances ;

- 15 tâches pour comprendre les documents iconographiques, l'élève est appelé à faire appel à ses propres connaissances.

Ces différentes tâches de l'élève tout au long du contenu de la Mécanique dans le manuel favorisent plus ou moins les apprentissages et développent des connaissances.

\section{DISCUSSION}

Nous discutons de l'effet des styles pédagogiques et les documents iconographiques sur l'apprentissage du contenu du savoir à enseigner de la partie Mécanique de physique dans le manuel intitulé Physique Chimie (Collection AREX, Les classiques africains) des classes de seconde scientifique. Notre échantillon est considéré comme représentatif d'une population moyenne d'étudiants en Master Professionnel de sciences physiques à l'Ecole Normale Supérieure d'Abidjan.

Nous notons que l'utilisation d'un style pédagogique type par l'enseignant pour enseigner les documents textuels et iconographiques d'une part; la lecture et la compréhension de ces documents par l'élève d'autre part, sont fondamentales dans l'apprentissage et la construction du savoir par l'élève. Nous articulons notre discussion autour des deux points suivants : les styles pédagogiques, et les documents textuels et iconographiques.

- Concernant les styles pédagogiques, le style pédagogique informatif prédomine avec un pourcentage de $41 \%$. Cette prédominance de ce style indique que les auteurs du manuel ne privilégient pas la participation active de l'élève dans la construction du savoir. Cela est 
vérifié par la faible problématisation et la très faible incitation à l'autonomie et à la réflexion dans les démarches utilisées par les auteurs pour faire passer le savoir à enseigner. En conséquence, il n'y a aucun développement du plaisir de l'invention chez l'apprenant ; pas de réflexion demandée, toutes les informations sur les notions sont mises à sa disposition. L’élève n'est donc pas engagé dans l'élaboration et la construction de son savoir.

Le style pédagogique injonctif (28\%) relevé à travers les différentes activités indique que les solutions sont imposées sans argumentation. L'information est transmise de manière dogmatique avec des consignes ou des directives à appliquer.

Ces deux styles pédagogiques $(41 \%+28 \%)$ largement utilisés tout au long de la partie Mécanique montrent que les tâches assignées aux élèves ne sont pas conçues pour les inciter à la création de nouveaux registres, et donc les connaissances qu'ils vont acquérir seront des connaissances superficielles donc inertes des notions et des concepts. Les conséquences de telles pratiques sont que les élèves ne pouvant pas mettre en relation les notions et les concepts sous-jacents vont se refuser dans la mémorisation pour réussir plutôt que de faire un transfert adéquat de ces connaissances dans la résolution de nouveaux problèmes. Cela va amener l'élève à appliquer de procédures mémorisées sans nécessairement comprendre la notion ou le concept (Ferguson \& Bodner, 2008)

- Concernant les documents textuels et iconographiques, nous notons que la majorité des documents textuels sont de type informatif (36/57): ils énoncent des faits et ne placent pas l'élève en situation de travail pour solliciter sa participation à la construction du savoir. Ces documents textuels sur lesquels le savoir à enseigner a été conçu n'impliquent pas activement l'élève et ne lui permettent pas d'acquérir les connaissances de manière vivante. L'esprit de l'élève reste en veilleuse car ces documents n'intriquent ni son esprit par des questions, ni l'obligent à trouver réponse à des problèmes. Ainsi, face à un contenu immense avec un rythme rapide du cours qui ne laisse pas le temps de faire des liens et de bien comprendre le "pourquoi », l'élève pour s'en sortir sera amené à chercher à apprendre des règles locales (principes, théorèmes, et lois) qu'il sortira souvent de leur contexte et les appliquera de manière inappropriée.

Quant aux documents iconographiques, nous notons qu'ils ont plus une fonction illustrative (35/57). La plupart des figures ne demandent pas à l'élève qu'il fasse une activité, il doit juste lire la figure. Leur prédominance montre qu'elles sont plutôt intégrées dans le contenu du manuel pour illustrer les textes et parfois faire comprendre certaines choses qui sont plus faciles à intégrer visuellement. Or, 
l'élève ne s'approprie véritablement un savoir que s’il est intégré dans un contexte social qui donne du sens à ce qu'il apprend, qui lui renvoie ses manques, les mises au point à faire, les décalages (Boulnois, 2008). Le manuel en le privant de tout cela, l'élève est inactif et intellectuellement moins engagé dans le processus. Ses acquisitions sont alors moins vivantes et moins solides.

Les documents textuels et les figures utilisés dans la partie Mécanique du manuel montrent que l'élève n'est pas activement et intellectuellement engagé dans le processus d'apprentissage. Le contexte des apprentissages n'est pas intégratif. Or on n'apprend, on ne progresse et on n'acquiert que par l'effort. Il faut que l'esprit se prenne à la chose, qu'elle l'intrigue, qu'elle le préoccupe ; qu'elle soit pour lui une question et un tourment ; qu'il déploie ses forces et ses ressources pour en venir à bout (Nguessan, 2016).

Pour construire le savoir, les auteurs doivent choisir des exemples significatifs et représentatifs mais en même temps la démarche doit être basée sur l'action dans le seul but d'apprendre à l'élève « à diriger son œil et son oreille, ses lèvres et sa bouche vers les matériaux qu'on lui présente, de façon à les graver dans sa mémoire pendant que, du même coup, ses images mentales se libèrent de ce travail mécanique, pour se porter vers ce qui est d'un intérêt vivant pour son organisme » (Savard, 1950). L'activité doit s'exercer sur la réalité, sur les choses et sur la vie ; si elle cantonne dans les cadres et dans l'atmosphère scolaires, elle ne sera jamais que formelle et verbale. Or ce sont justement les choses qui excitent la curiosité et l'intérêt de l'élève; c'est à propos des choses qu'il se pose des problèmes de manipulation, qu'il cherche des explications et qu'il éprouve le besoin de nommer; c'est au contact des choses que son esprit est actif. Ce qu'il apprend doit être vivant et organique. L'enseignant doit organiser l'acquis, le coordonner et le développer.

Une telle démarche ne laissera plus de place au dogmatisme et à la passivité mais plutôt au développement spontané de l'intelligence des élèves qui stimulent les forces intelligentes de chacun et les poussent à l'épanouissement; par des méthodes qui font des esprits curieux, ouverts sur la vie et le monde, des esprits justes, méthodiques, réfléchis, soucieux de vérification (Delannoy-Courdent, 2011). L'élève doit donc être acteur de son enseignement pour favoriser ses apprentissages.

\section{CONCLUSION}

Nous pouvons maintenant revenir en arrière et embrasser du regard le chemin que nous avons parcouru. L'intérêt, que nous manifestons, des fins que se proposent les auteurs du manuel intitulé Physique Chimie (Collection AREX, Les classiques africains) des classes de seconde scientifique, des 
moyens auxquels ils recourent pour assurer son action, des méthodes qui y sont utilisées pour faire acquérir les connaissances, est en deçà de nos attentes.

A la lumière des résultats de cette étude, nous notons que ce manuel ne peut constituer un livre de référence ni pour l'enseignant, ni pour l'élève. En effet, le manuel intitulé Physique Chimie (Collection AREX, Les classiques africains) des classes de seconde scientifique tel qu'il est privilégie plus l'usage du style pédagogique informatif et celui des figures illustratives où les démarches utilisées par les auteurs pour faire passer le savoir à enseigner sont caractérisées par la faible problématisation et la très faible incitation à l'autonomie et à la réflexion. L'exposé des savoirs et les documents proposés sont davantage conçus pour favoriser le dogmatisme et la passivité de l'élève.

Il parait donc essentiel d'intégrer dans la formation continue de l'enseignant, une formation didactique basée sur la compréhension des textes et des styles pédagogiques pour soutenir une pédagogie constructiviste en classe. Ainsi, l'enseignant pourra former et accompagner les élèves dans la maîtrise des environnements didactiques; utiliser l'accompagnement personnalisé pour promouvoir des activités méthodiques concernant l'utilisation du manuel en vue de favoriser la construction du savoir et surtout le transfert des connaissances. Il s'agit de faire des élèves des personnes de parler de tout, à la conservation nourrie et brillante ; possédant des esprits curieux, ouverts sur la vie et le monde, des esprits justes, méthodiques, réfléchis, soucieux de vérification ; capables de la maîtrise des lois générales que sont : celles de l'association des images et des idées, celles de la mémoire, celles de l'abstraction et du raisonnement, celles de la raison, celles de la volonté libre et de la conscience.

\section{References:}

Bernard, S., Clément, P Carvalho, G.S. (2007). "Méthodologie pour une analyse didactique des manuels scolaires, et sa mise en œuvre sur un exemple ». In: Le manuel scolaire d'ici et d'ailleurs, d'hier à demain (Coord : M. Lebrun), Presses de l’Université du Québec (CD).

Boulnois, O. (2008). Au-delà de l'image: Une archéologie du visuel au Moyen-âge, V-XVI siècle.

Clément, P. (1998). « La biologie et sa didactique. Dix ans de recherches », Aster, vol. 27, p.57-93.

Clément, P. (2004). "Science et idéologie : exemples en didactique et épistémologie de la biologie », dans Actes du colloque Sciences, médias et société, Lyon, ENS-LSH, p.53-69.

Delannoy-Courdent, A. (2011). «Les procédés de diffusion de l'enseignement et la vulgarisation scientifique : des témoins de stratégies, 
des vecteurs de contenus " in Transmettre les sciences: vulgarisation et enseignement, Spirale 48, Université Lille 3.

Ferguson, R. \& Bodner, G.M. (2008). "Making sense of the arrow-pushing formalism among chemistry majors enrolled in organic chemistry", Chemistry Education Research and Practice, 9, 102-113.

Hannoun, P. (2008). « La mécanique vue par les sciences de l'ingénieur et la physique. Des ancrages théoriques communs, des éclairages différents; le cas du frottement». Dans A. Hasni et J. Lebeaume (dir.), Interdisciplinarité et enseignement scientifique et technologique (p. 111129). Lyon : INRP. Sherbrooke (Québec) : Éditions du CRP.

Lasswell, H. D. (1952). «L'analyse de contenu et le langage de la politique » in Revue française de science politique, vol. II, $\mathrm{n}^{\circ} 3$; p.275-288.

Nanzouan, S.P., N'dri, K., Tiamaoui, B.T. (2000). Physique Chimie. Seconde Scientifique. Collection AREX, Les classiques africains. Old Moka Road, Bell Village, Port Louis Mauritius.

Nguessan, K. (2016). «Analyses didactique et épistémologique des aides didactiques dans le manuel de Physique Chimie (Collection AREX) des classes de premières scientifiques de Côte d'Ivoire, cas de la notion d'énergie » In International Journal Current Research, Vol. 8, Issue 04, pp. 29147-29160.

Savard, G. 1950. Pédagogie contemporaine. Librairie DELAGRAVE. Paris. 